PHYSICAL REVIEW D 95, 079902(E) (2017)

\title{
Erratum: Gluonic transversity from lattice QCD [Phys. Rev. D 94, 014507 (2016)]
}

\author{
W. Detmold and P. E. Shanahan \\ (Received 23 March 2017; published 7 April 2017)
}

DOI: 10.1103/PhysRevD.95.079902

On further investigation, it was discovered that the decomposition of the parton distribution functions of the spinindependent gluon operator in Eq. (37) of the main paper is incorrect. This lead to a number of errors, which are corrected here.

Replacing Eq. (32) of the main paper, the gluonic analogue of the Soffer bound, for spin-1 particles, is [1-4]

$$
|\delta G(x)| \leq \frac{1}{2}\left(f_{1}(x)+\frac{1}{2} f_{1 L L}(x)+g_{1}(x)\right),
$$

where $\delta G(x)$ is the gluonic transversity distribution defined in Eq. (14) of the main paper, $f_{1}(x)$ and $f_{1 L L}(x)$ are the spinindependent gluon distributions, and $g_{1}(x)$ is the spin-dependent gluon distribution. The notation here for $f_{1}, f_{1 L L}$, and $g_{1}$ is the same as in Refs. $[3,4]$, while $\delta G(x)$ is named $h_{1 T T}$ in those works.

Replacing Eq. (36) of the main paper, the analogue of the Soffer bound for the leading Mellin moments of gluon distributions is [4]

$$
\left|A_{2}\right| \leq \frac{1}{24}\left(5 B_{2,1}-6 B_{2,2}\right),
$$

where $A_{2}$ is the reduced matrix element defined in Eq. (10) of the main paper and $B_{2,1}$ and $B_{2,2}$ are linear combinations of the moments of the structure functions $f_{1}$ and $f_{1 L L}$ in Eq. (1), defined through

$$
\left\langle p E^{\prime}\left|\overline{\mathcal{O}}_{\mu_{1} \mu_{2}}\right| p E\right\rangle=S\left[M^{2} E_{\mu_{1}}^{\prime *} E_{\mu_{2}}\right] B_{2,1}\left(\mu^{2}\right)+S\left[\left(E \cdot E^{\prime *}\right) p_{\mu_{1}} p_{\mu_{2}}\right] B_{2,2}\left(\mu^{2}\right) .
$$

This equation replaces Eq. (37) from the main paper.

Several figures must also be replaced. Figures 1 and 2 below replace Figs. 6 and 8 from the main paper. The conclusions of the analysis, including that the gluon Soffer bound in the spin-1 $\phi$ meson is saturated to approximately $80 \%-100 \%$, do not change.
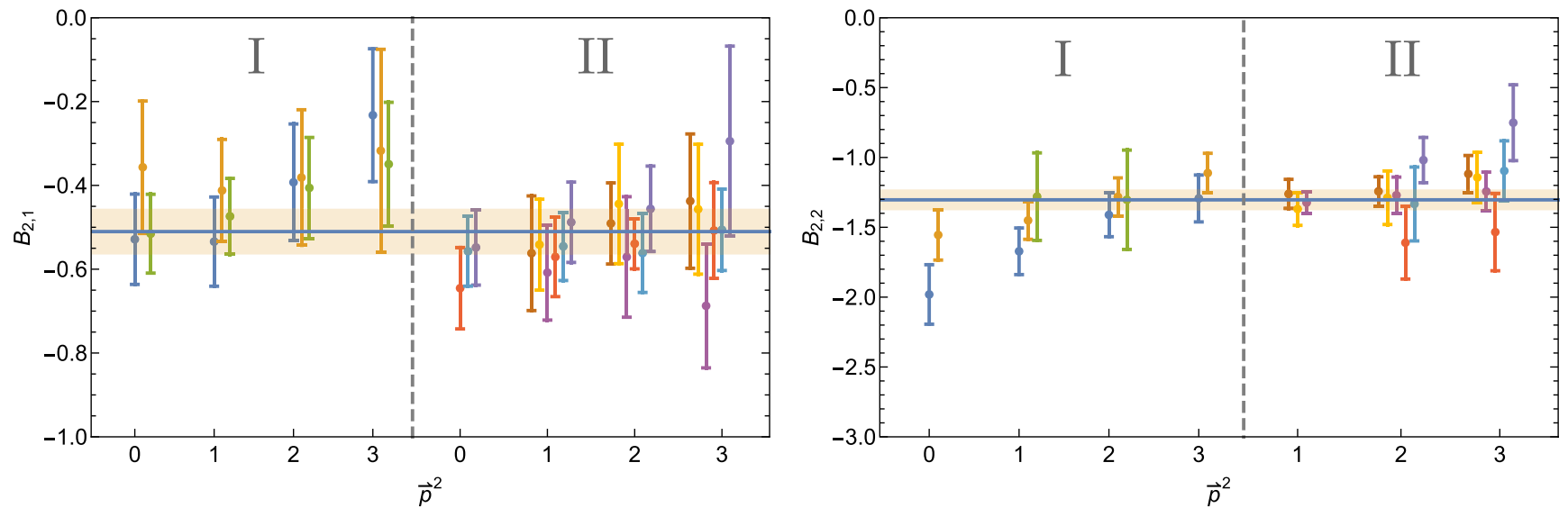

FIG. 1. Reduced matrix elements $B_{2,1}$ and $B_{2,2}$ extracted from ratios of two- and three-point functions as described in Secs. III C and IV of the main text. Results in sections I and II of the figure are determined using vectors in the $\tau_{1}^{(3)}$ and $\tau_{3}^{(6)}$ representations. Different colors (offset on the horizontal access for clarity) denote different basis vectors. These results can be compared with those in Fig. 2 with Wilson-flowed gauge fields. The horizontal bands are fits shown to guide the eye. 


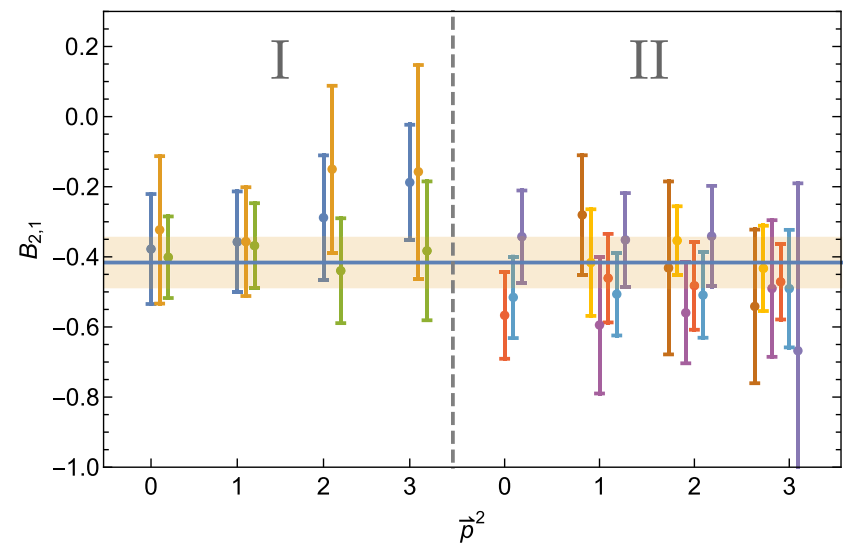

(a) Two steps of HYP smearing

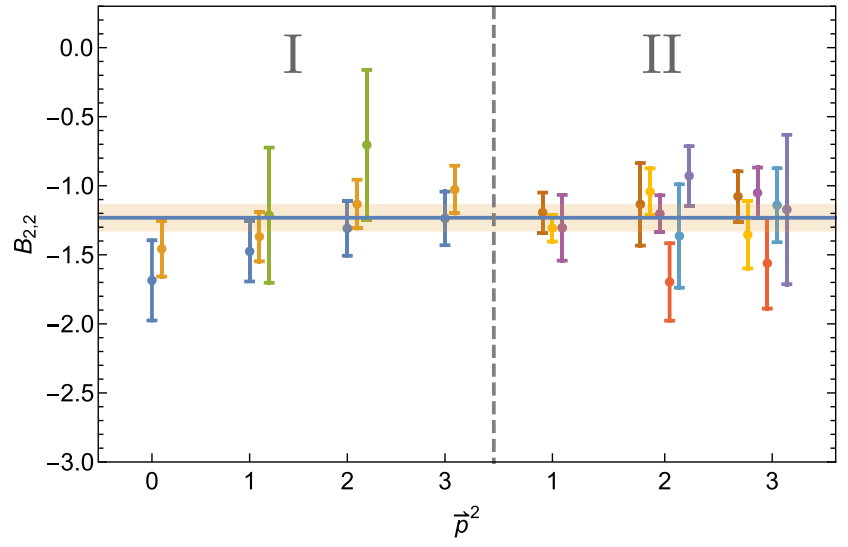

(c) Two steps of HYP smearing

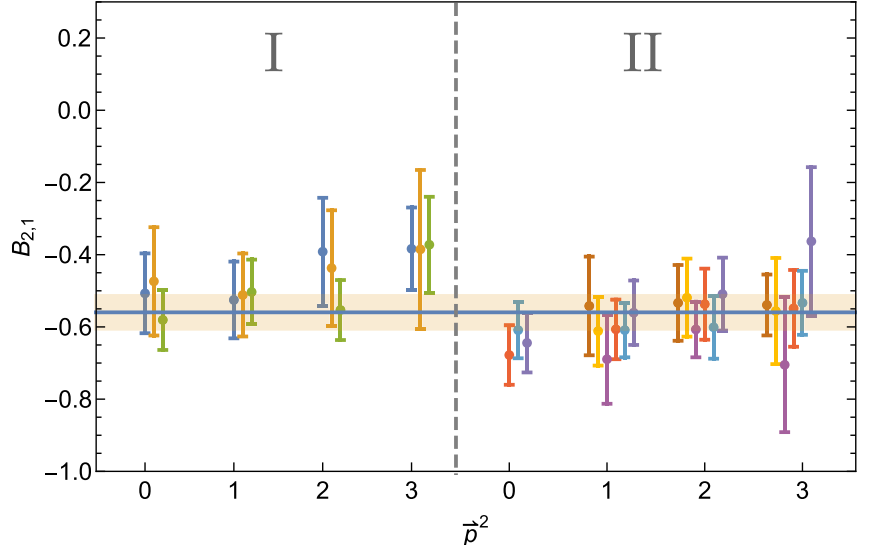

(b) Five steps of HYP smearing

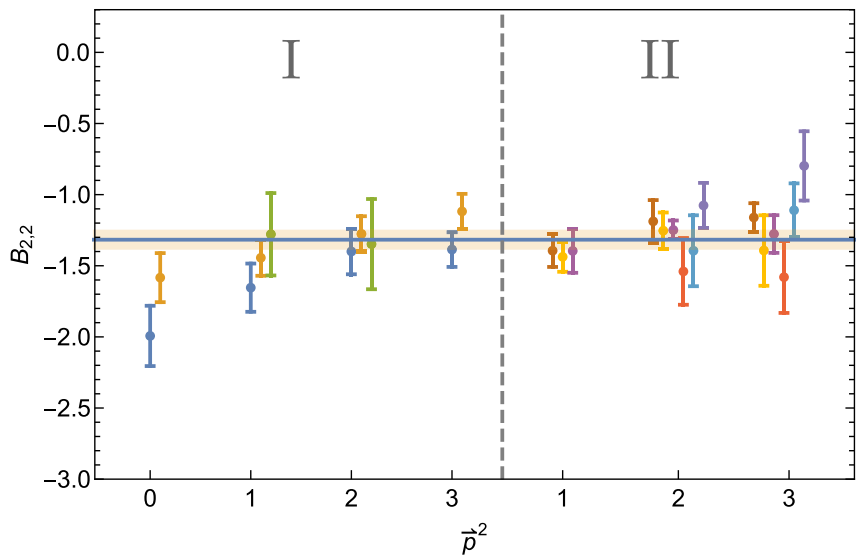

(d) Five steps of HYP smearing

FIG. 2. Reduced matrix elements $B_{2,1}$ and $B_{2,2}$ extracted from ratios of two- and three-point functions as described in Secs. IIIC and IV of the main text. Wilson flow [5] was applied to the links in the gluon operator as described in the text. Results in sections I and II of the figure are determined using vectors in the $\tau_{1}^{(3)}$ and $\tau_{3}^{(6)}$ representations. Different colors (offset on the horizontal access for clarity) denote different basis vectors. The horizontal band is a fit shown to guide the eye.

[1] X. Artru, M. Elchikh, J.-M. Richard, J. Soffer, and O. V. Teryaev, Phys. Rep. 470, 1 (2009).

[2] R. Jaffe (private communication).

[3] D. Boer, S. Cotogno, T. van Daal, P. J. Mulders, A. Signori, and Y.-J. Zhou, J. High Energy Phys. 10 (2016) 013.

[4] S. Cotogno, Few-Body Syst. 58, 92 (2017).

[5] M. Lüscher, J. High Energy Phys. 08 (2010) 071; 03 (2014) 092(E). 